\title{
Дискурсивный сетевой анализ: дискурсивно-динамический аспект исследования публичной политики
}

\author{
А.И. Корюшкин \\ Санкт-Петербургский государственный университет, \\ Россия, 199034, г. Санкт-Петербург, Университетская набережная, 7/9 \\ Email: a.koryushkin@spbu.ru
}

\begin{abstract}
Аннотация. Объектом анализа статьи является концепт дискурсивного сетевого анализа, предложенный британским политологом Филипом Лейфелдом, и, прежде всего, его сущностные характеристики, позволяющие оценить объяснительный потенциал такого концепта, представляющего собой комбинацию качественного контент-анализа и количественного сетевого анализа. Здесь интерпретируется принципиальная важность определения в этом концепте публичных политических дебатов в качестве динамичных дискурсивных сетей, дискутирующих проблемы конкретных политических курсов в их развитии, детальной творческой разработки в нем центрального понятия «политический дискурс», подчеркивающей его динамическую природу, также значимость объединения в его методологии актор-ориентированных и контенториентированных подходов к изучению политических дискурсов конкретных политических курсов, что позволяет концептуализировать и замерять ко-эволюцию акторов и их контентов в процессе динамичных изменений выработки, реформирования и смены политических курсов, обеспечивая возможность их долгосрочного мониторинга.
\end{abstract}

Ключевые слова: дискурсивный сетевой анализ, публичные политические дебаты, политический дискурс, качественный контент-анализ, количественный сетевой анализ, динамический аспект исследования, публичная политика.

Благодарности: работа выполнена при поддержке Российского фонда фундаментальных исследований, грант № 18-011-00705 «Объяснительный потенциал сетевой теории в политических исследованиях: методологический синтез как аналитическая стратегия».

Для цитирования: Корюшкин А.И. 2020. Дискурсивный сетевой анализ: дискурсивнодинамический аспект исследования публичной политики. Via in tempore. История. Политология, 47 (4): 921-932. DOI: 10.18413/2687-0967-2020-47-4-921-932.

\section{Discurse network analysis: discursive-dynamic aspect of public policy research}

\author{
Alexander I. Koryushkin \\ Saint Petersburg State University, \\ 7/9 University Embankment, St. Petersburg, 199034, Russia \\ Email: a.koryushkin@spbu.ru
}

\begin{abstract}
The paper's analysis is aimed at the concept of discourse network analysis suggested by the British political scientist Philip Leifeld and, most of all, at its essential features that make it possible to evaluate an explanatory potential of such a concept that appears to be a combination of qualitative content-analysis and quantitative network analysis. It is interpreted here a principal importance of defining in this concept of the public political debates as dynamic discourse networks discussing policy problems in their development and of the detailed and creative elaboration of the central notion of «political discourse» emphasizing its dynamic nature as well as a significance of combining in its methodology of the actor-oriented and the content-oriented approaches to the research over policy
\end{abstract}


discourses that let conceptualizing and measuring co-evolution of actors and their contents in the process of dynamic changes of formulation, reformation and shift of the policies thus having provided an option of their long-term monitoring.

Keywords: discourse network analysis, public political debates, political discourse, qualitative contentanalysis, quantitative network analysis, dynamic aspect of research, public policy.

Gratitudes: This work was supported by the Russian Foundation for Basic Research, Grant No. 18-01100705 «The Explanatory Potential of Network Theory in Political Research: Methodological Synthesis as an Analytical Strategy».

For citation: Koryushkin A.I. 2020. Discurse network analysis: discursive-dynamic aspect of public policy research. Via in tempore. History and political science, 47 (4): 921-932 (in Russian). DOI: $10.18413 / 2687-0967-2020-47-4-921-932$.

Современное постиндустриальное информационное общество на этапе его неуклонно возрастающей и углубляющейся цифровизации все более очевидным образом обнаруживает себя в качестве масштабного сетевого явления, представляющего собой глобальную сеть сетей потоков информации. Оно является предметом социально-научных исследований, в рамках которых разработан и применяется обширный инструментарий политического сетевого анализа, особенно важного в изучении таких центральных, фокусных политических явлений, как процессы принятия политических решений. Сетевая публичная политика, разворачиваясь в интегрированном с интернет-средой публичном пространстве, «представляет собой одновременно процесс и результат взаимодействия широкого круга разнообразных социально-политических акторов, использующих сетевые ресурсы и технологии для выработки способов решения проблем общественной значимости и их интеграции в управленческую практику» [Мирошниченко, Морозова, 2017, с. 83]. Вместе с тем в публичной сфере как информационно-коммуникативном пространстве происходит конструирование групп общественности, участвующих в формировании общественно-политического дискурса. Здесь «конституируются и институционализируются различные политические акторы - участники делиберативного политического процесса, связывающего дискурсивные практики с циклическим производством публичных решений на основе диалога и согласования позиций сторон. На этой основе формируется деятельностно-активистский потенциал или институциональное измерение публичной политики, характеризуемое эффективностью публичной деятельности акторов и их участия в принятии решений» [Там же, с. 85]. Обе эти ипостаси современного политического процесса, его сетевой характер и его дискурсивную природу, соединяет в себе дискурсивный сетевой анализ публичной политики, который представляет собой синтетический эмпирико-теоретический инструмент исследования сетевых и дискурсивных параметров процесса выработки, изменения и смены конкретных политических курсов (policies). Предметом же нашего исследования является получающий все более широкое признание в научном сообществе вариант дискурсивного сетевого анализа, разработанный британским политологом Филипом Лейфелдом, с целью анализа его наиболее важных характеристик, позволяющих интерпретировать его объяснительный потенциал.

В недавнем авторитетном издании «The Oxford Handbook of Political Networks» [Victor et al., 2017] Филип Лейфелд предложил трактовку публичных политических (policy) дебатов в качестве динамичных сетей, исследуемых им в рамках дискурсивного сетевого анализа [Leifeld, 2017]. Политические дебаты, или политические дискурсы, представляют собой вербальные взаимодействия между политическими акторами в отношении того или иного политического курса (policy). Политические акторы делают публичные заявления относительно того, какие политические инструменты, меры они считают полезными и какие они отвергают. Целями и задачами такого участия акторов в политических 
дебатах являются (а) доведение до избирателей или потенциальных союзников своего представления о предлагаемом политическом курсе, (б) убеждение других акторов в правильности предлагаемых им мер по разработке и реализации данного курса, и (в) понижение уровня его собственной неопределенности в отношении такого курса посредством получения необходимых знаний от других политических акторов.

Такие дебаты очень важны по ряду оснований. Во-первых, в их ходе определяется, какие политические курсы (policies) включаются в повестку дня парламентских заседаний, а какие не попадают в нее. Во-вторых, они часто оказывают влияние на общественное мнение, которое затем тем или иным образом отражается и в сфере принятия политических решений. В-третьих, они играют также роль и в менее публичных формах взаимоотношений между политическими акторами, в рамках дискуссий и процессов принятия решений за закрытыми дверями или в бюрократической сфере. Кроме того, такие публичные дебаты обладают, посредством изменения в их ходе восприятия акторами проблем, потенциалом влияния на способы решения поставленных в политическом курсе (policy) задач для достижения его результатов еще до его реализации. Политические дебаты, будучи одной из форм политического дискурса, являются сетевым феноменом, поскольку публичные заявления политических акторов, обладающие свойствами реляционного действия, зависят друг от друга во временном и общественно-пространственном плане и могут исследоваться средствами политического сетевого анализа. Целью политического сетевого анализа является описание структур политического дискурса и логическое выведение (inferentiation) порождающих их процессов на базе инструментария сетевого анализа [Leifeld, 2017].

В своей более ранней работе Ф. Лейфелд [Leifeld, 2010] обосновывает политический дискурсивный анализ в качестве важного отсутствующего звена в изучении политически (policy) ориентированного дискурса. Если сам политический дискурс можно определить как формальный обмен обоснованными взглядами относительно того, какие из нескольких альтернативных политических курсов следует применить для разрешения той или иной социально-политической проблемы [Johnson, Johnson, 2000], то его исследованию посвящен обширный массив научной литературы. Однако, пишет Ф. Лейфелд, несмотря на обилие теоретических и эмпирических разработок такого рода, имеет место разрыв, своеобразный провал между контент-ориентированными и акторориентированными подходами в изучении политического дискурса. Этот провал он предлагает заполнить на основе новой методологии эмпирического и теоретического анализа политического дискурса посредством концепта дискурсивного сетевого анализа, представляющего собой комбинацию качественного контент-анализа и количественного социально-сетевого анализа.

Действительно, в изучении политического дискурса элиты представлены два существенно отличающихся подхода. Один из них исследует содержание дискурса, задаваясь вопросами о том, «какие существуют конкурирующие фреймы?», «как они связаны с политическими целями и властными отношениями?» или «какие понятия дискурса связаны друг с другом?». Такого рода контент-ориентированный подход типичен в критическом дискурсивном анализе [Wodak, Meyer, 2009], в семантическом сетевом анализе [Brandes, Corman, 2003; Popping, 2003] и в анализе дискурсов легитимности [Nullmeier, Wrobel, 2005; Hurrelmann et al., 2009]. В противоположность ему другой, актор-ориентированный подход к изучению политического дискурса имеет дело с конфигурациями политических (policy) субсистем, выделяемых согласно предпочтениям и идеям акторов. Он сконцентрирован на изменениях взаимосвязей между акторами, которые могут в конечном счете объяснять изменения или смену политических курсов. Примерами таких акторориентированных подходов являются концепция коалиций давления [Sabatier, Weible, 2007], теория прерывающегося равновесия [Baumgartner, Jones, 1991], подходы, ориентированные на изучение эпистемных сообществ [Haas, 1992] и парадигм политических кур- 
сов [Hall, 1993]. Наиболее типичным примером такого рода актор-ориентированных подходов является концепция коалиций давления (advocacy coalitions), в рамках которой акторы делятся на две противостоящие друг другу коалиции. При этом сущностные убеждения акторов относительно того или иного политического курса (policy core beliefs) являются своеобразным «клеем», скрепляющим коалиции воедино [Sabatier, Weible, 2007]. Taкие сущностные убеждения имеют больше сходств внутри коалиций, чем между коалициями. В то же время члены этих коалиций предпринимают непрерывные попытки убедить своих оппонентов в правильности своих позиций, и это взаимодействие между коалициями и составляет содержание политического дискурса. Если внешние события приводят к переосмыслению таких сущностных убеждений и изменению «членства» ключевых акторов в коалициях, то реформирование политического курса (policy) становится возможным.

Что же касается связи между акторами и их концептами, то она представляет для обоих подходов к изучению политического дискурса побочный, вторичный интерес. Они не анализируют ко-эволюцию конфигураций акторов и идей систематическим образом, что Ф. Лейфелд полагает задачей предлагаемого им нового, сущностно реляционного варианта дискурсивного сетевого анализа, объединяющего качественный контент-анализ и количественный сетевой анализ, и призванного концептуализировать и замерять коэволюцию акторов и их концептов в процессе их динамических изменений. Он является средством наблюдения и измерения дискурса и может объединяться со многими теоретическим концептами, лежащими в основе политического дискурса, включая концепты дискурсивных коалиций, высказывания политических заявлений, дискурсов легитимности, коалиций давления и эпистемных сообществ. Этот подход тесно связан и может быть интегрирован с сетевой концепцией политических курсов и может интерпретироваться как расширение политического сетевого анализа за счет включения в него дискурсивных структур [Leifeld, 2010, pp. 2-4].

Роль дискурса в объяснении процессов публичной политики и разработки конкретных политических курсов (policies) явилась предметом исследований в политической науке еще в конце прошлого века в рамках аргументативного подхода. Исходным, базовым положением этого подхода является представление о том, что социальные акторы, противостоящие друг другу в политическом конфликте, следуют различным модусам рациональности [Clegg, 1989], в основе которых обнаруживаются различные рамки, фреймы интерпретации, используемые различными конфликтующими группами. Такие фреймы состоят из интер-субъективных структур смысла, состоящих из наборов идей, концептов и категорий и придающих смысл социальной реальности и задающих способы ее преобразования. В литературе по интерпретативному подходу группы акторов, участвующие в разработке публичной политики и являющиеся носителями относительно одинаковых фреймов интерпретации, определяются в качестве дискурсивных коалиций [например, Hajer, 1993, 1996, 2002; Fischer, Forester, 1993; Atkinson, 2000; Szarka, 2004]. Такие дискурсивные коалиции принимают участие в борьбе за дискурсивную гегемонию, а сама эта борьба за институционализацию своего дискурса является, по утверждению ряда авторов, сутью политики [Sederberg, 1984; Laclau, Mouffe, 1985].

Такая разработка аргументативного поворота в политическом анализе предопределила широкое распространение в литературе кейсов, применяющих такие формы качественного дискурсивного анализа, как нарративный анализ, метафорический анализ и фрейм-анализ [Linder, 1995; Lenschow, Zito, 1998; Atkinson, 2000; Szarka, 2004; Falk, 2007; Ferrari, 2007], и позволяющих осуществлять глубинное исследование широкого круга политико-дискурсивных процессов и противоречий [Yanow, 2000; Bryman, 2012]. Вместе с тем, как отмечает А. Мюллер [Muller, 2015], эти методы ограничены в их применении в сравнительном исследовании дискурсивных коалиций, поскольку, не давая возможности получения никаких объективных стандартизированных измерений интенсивности политических конфликтов и уровня поляризации между дискурсивными коалициями, не дают 
представление о мере, в которой одна из таких коалиций доминирует в дискурсивном поле, в то время как такое дискурсивное доминирование может быть одним из определяющих факторов в исходе политических конфликтов [Leifeld, Haunss, 2012]. Надо полагать, именно в свете такой или подобной критики Ф. Лейфелд обратился к анализу феномена поляризации дискурсивных коалиций, предложив интерпретацию и анализ публичных политических дебатов как политических дискурсов, обладающих сложным комплексом свойств, которые он определяет как семь их сущностных характеристик.

Bo-первых, полагает он [Leifeld, 2014], политический дискурс динамичен, поскольку его акторы неоднократно принимают участие в политических дебатах относительно того или иного политического курса, и скорее их следующие в ходе дебатов один за другим заявления, чем их одномоментные утверждения, составляют сущность такого дискурса.

Во-вторых, политический дискурс реляционен, так как ни его акторы не изолированы в ходе дебатов друг от друга, ни их заявления не распространяются в политическом пространстве и временном интервале обсуждения конкретного политического курса (poliсу) лишь случайным образом. Здесь имеет место взаимодействие между акторами, зачастую апеллирующими к тому, что было прежде высказано в ходе дебатов другими акторами, поэтому к исследованию такого взаимодействия вполне применим инструментарий социального сетевого анализа, позволяющего эффективно анализировать взаимозависимость и потоки взаимовлияния друг на друга индивидов, групп и институтов.

B-третьих, мотивация акторов к участию в таком дискурсе основана на неопределенности позиции других акторов. Политический дискурс заключает в себе как властные, так и инструментально-альтернативные компоненты разработки оптимального политического курса. Базовым основанием возникновения и существования такого политического дискурса является неопределенность относительно того, приведет ли данный политический курс к достижению необходимого для разрешения той или иной проблемы результата и каковы будут его последствия. С другой стороны, сама эта неопределенность используется акторами, за которыми стоят определенные существенные интересы. Делая в ходе дискурса конкретные заявления или выдвигая те или иные требования, организации и институты пытаются убедить неопределившихся согласиться с их требованиями, в результате чего «движущей силой» такого дискурса является определенная цель, а сам дискурс может быть истолкован в терминах властных отношений.

B-четвертых, поскольку дискурс основан на динамичных нерандомных взаимодействиях между акторами, он обычно обладает свойством пат-зависимости (pathdependence), так как акторы редко выдвигают в ходе дискурса совершенно новые идеи. Если бы все заявления и утверждения акторов, участвующих в дискурсе, были бы основаны на абсолютно новых идеях, стимул к участию в таком дискурсе исчезал бы изначально, потому что невозможно было бы достичь в его процессе каких-либо целей.

В-пятых, дискурс всегда фрагментирован и поляризован. Политическое пространство разработки конкретных политических курсов и, соответственно, их дискурсов состоит из нескольких конкурирующих коалиций акторов. Эти коалиции давления (advocacy coalitions) или дискурсивные коалиции, как правило, вновь и вновь выдвигают когезионные наборы убеждений или утверждений относительно того или иного политического курса, но при этом взаимное пересечение таких наборов убеждений и утверждений относительно мало. Такое явление может отражаться в понятии социального баланса, обнаруживаемого в математических и компьютерных «моделях культурной сегрегации и поляризации».

B-шестых, исследования, проводимые в рамках концепции коалиций давления (advocacy coalitions), свидетельствуют о том, что как внутри коалиций, так и между ними имеет место процесс взаимного узнавания, научения (learning process) акторами друг друга. А это означает, что коалиции, участвующие в дискурсе, будучи фрагментированы или поляризованы, не полностью изолированы друг от друга. 
В-седьмых, политический дискурс обладает свойством неравновесности. При том понимании, что участие в дискурсе является одним из средств достижения целей, определяемых в том или ином конкретном политическом курсе, или средством понижения уровня неопределенности относительно возможных в будущем проблем, дискурс продолжает разворачиваться во времени до тех пор, пока цели почти всех участвующих в нем индивидов не будут достигнуты. Поскольку такое равновесие редко возникает в политике, политический дискурс в качестве неравновесного явления обладает, вероятно, неограниченным горизонтом времени. Если бы такое равновесие здесь было, то каждый из акторов дискурса вновь и вновь высказывал бы в определенном порядке один и тот же набор идей, или же дискурс прекратился бы, поскольку прежние противоречия между антагонистами были бы разрешены [Leifeld, 2014, pp. 1-2].

Приведенная здесь формулировка и обстоятельная, творческая интерпретация Ф. Лейфелдом содержательных характеристик феномена политического дискурса представляется важным этапом в разработке концепции дискурсивного сетевого анализа как эффективного инструмента исследования проблем разработки и реализации конкретных политических курсов (policies) в их политической динамике. Вместе с тем весьма значимым моментом в дальнейшей разработке этой концепции явилась совместная статья Филипа Лейфелда и Лоуренса Бранденбергера [Leifeld, Brandenberger, 2019], предложившая объяснение процессов формирования и поддержания единства участвующих в политических дебатах эндогенных коалиций на основе разделяемых убеждений в качестве дискурсивных коалиций в рамках дискурсивного сетевого анализа. При этом они анализируют процесс познания акторами политических (policy) убеждений друг друга и утверждают, что возникающие при этом дискурсивные коалиции формируются тремя различными способами.

Во-первых, полагают эти авторы, так как участвующие в сложном процессе выработки конкретных политических курсов акторы обнаруживают себя в ситуации неопределенности и даже риска, они на основе связывающих их взаимоотношений заимствуют, приобретают знания (learn) других акторов. Таким образом, возникают коалиции, которые формируются и сохраняются в качестве таковых в процессе обретения знаний о политических (policy) убеждениях акторов, которые демонстрировали подобные политические убеждения относительно того или иного политического курса в прошлом. В этом случае известная в литературе по коллаборативному политическому управлению «гипотеза риска» (Berardo, 2014; Berardo and Lubell, 2016) распространяется на процессы формирования убеждений и коалиций. Наблюдая процесс обсуждения того или иного политического курса и обнаруживая акторов такого обсуждения с весьма похожими убеждениями, акторы идентифицируют «соседей» по коалиции. Такой механизм формирования коалиций авторы называют механизмом позитивной взаимности и приводят на микро-уровне эмпирические свидетельства в пользу его значительной роли в процессе формирования коалиций.

Во-вторых, в дополнение к таким сильным понятийным связям, акторы обнаруживают аргументы в пользу того или иного политического курса посредством того, что авторы называют «слабыми связями». Для чего необходимо, чтобы (а) большое число других акторов выражали вполне определенную позицию по обсуждаемому политическому курсу (policy) и (б) мнения многих из этих других акторов были хотя бы в минимальной степени идеологически совместимы с мнением такого «фокусного» актора по другим проблемам или политическим курсам и потому заслуживали бы принятия, признания этим актором в качестве относительно правомерных. Таким образом, акторы стремятся к установлению взаимоотношений не только посредством механизма позитивной взаимности. Они также ориентированы на обретение в результате установления таких «слабых связей» новых для них убеждений, так или иначе совместимых с их собственными (Berardo and Scholz 2010), в результате чего формирование коалиций происходит посредством обнаружения акторами по меньшей мере относительного соответствия их систем убеждений. Такой, второй механизм формирования коалиций авторы называют механизмом инновационного познания. 
В-третьих, акторы, делая выводы и из негативных случаев обсуждения предыдущих политических курсов (policies), когда они имели в ходе политических дебатов по ним существенные конфликты с другими акторами, в большей степени склонны оппонировать политическим убеждениям и доводам таких акторов. Такое усиление конфликтных взаимоотношений порождает взаимное отталкивание между коалициями, дополняя действие механизма позитивной взаимности и повышая уровень соответствия внутри коалиций. Такой механизм формирования коалиций авторы называют механизмом негативной взаимности.

Эти три механизма формирования коалиций дополняют друг друга, обеспечивая (a) повышение в результате взаимного усиления позитивных взаимоотношений уровня соответствия внутри коалиций, (б) перекрестный обмен инновационными доводами и аргументами в пользу того или иного политического курса между коалициями и (в) эффект отталкивания между коалициями в результате усиления конфликтных взаимоотношений. Ф. Лейфелд и Л. Бранденбергер уже более девяти лет (с ежедневной фиксацией) тестируют описанный здесь триединый процесс формирования эндогенных дискурсивных коалиций, используя современный динамический сетевой анализ реальных дебатов по конкретным политическим курсам, и на микро-уровне обнаруживают убедительные свидетельства в пользу такого его истолкования (Leifeld and Brandenberger, 2019).

В основании такого динамического сетевого анализа присутствуют следующие три процедуры: (1) идентификация политических акторов, выступающих в средствах массовой коммуникации с теми или иными утверждениями; (2) кодирование и связывание их с конкретными утверждениями, в которых каждый из них позитивным или негативным образом высказывает свои предпочтения относительно того или иного политического курса и (3) дифференцирующее определение таких утверждений по (а) актору, (б) концепту и (в) бинарной информации о поддержке или оппозиции (Leifeld, 2013). В некотором смысле этот метод может быть интерпретирован как своего рода кластерный анализ агрегированных аффилированных (партнерских) сетей, в котором анализ еще существенным образом сфокусирован на содержании утверждения. Но, как только такое утверждение возникает в средствах массовой коммуникации, в нем усматривается определенный актор и конкретный концепт. При этом такие сетевые данные визуализируются и анализируются при помощи программного обеспечения сетевого анализа с открытым исходным кодом Gephi 0.9.2.

Свидетельством роста авторитета и влияния в научном сообществе дискурсивного политического анализа явился тот факт, что второй, июньский номер 2020 года журнала «Politics and Governance» целиком посвящен дискурсивному сетевому анализу в его применении к исследованию дискурсивно-сетевых процессов публичной политики. Этот номер журнала, в роли научного редактора которого выступил Филип Лейфелд, имеет тематическое название «Политические дебаты и дискурсивный сетевой анализ» и содержит одиннадцать статей, раскрывающих содержание конкретно-эмпирических исследований разнообразных политических дебатов как дискурсивных сетей, демонстрирующих широту применения дискурсивного сетевого анализа и анализирующих его методологические качества, позволяющие получить значимый научный результат. Так, Schaub и Mertz [Schaub, Mertz, 2020] проводят сравнение политических сетей конкретных политических курсов и дискурсивных сетей, функционирующих в контексте экологической политики Германии в отношении микрозагрязнителей водоемов. Оба типа сетей, обнаруживая сравнимую структуру, обладают разным составом акторов, более широким в случае дискурсивных сетей за счет участия в них лидеров общественного мнения как внешних политических акторов. Kukkonen и Ylä-Anttila [Kukkonen, Ylä-Anttila, 2020], используя дискурсивный сетевой анализ для исследования научной политики Финляндии в рамках экологической политики в отношении климата, фокусируют свое внимание на специфической группе акторов, ученых и их участии в процессах обсуждения и разработки конкретных направлений экологической публичной политики. Ghinoi и Steiner [Ghinoi, Steiner, 2020] применяют дискурсивный сетевой анализ другой специфической группы акторов, законодателей 
различных политических партий и их участие в политических дебатах по климату в парламенте Италии. Эти и другие авторы статей, опубликованных в этом номере журнала, отмечают высокий методологический статус анализируемого нами варианта разработки дискурсивного сетевого анализа и его эвристический характер.

Подводя итоги проведенного в статье анализа важнейших характеристик предложенного Ф. Лейфелдом концепта дискурсивного сетевого анализа, следует признать продуктивность и теоретическую значимость интерпретации публичных политических дебатов как динамичных сетей, исследуемых в рамках дискурсивного сетевого анализа. Целью такого политического сетевого анализа является описание структур политического дискурса и логическое выведение порождающих их процессов выработки конкретных политических курсов. Усматривая разрыв, отсутствие важного звена в изучении политических курсов между контент-ориентированными и актор-ориентированными подходами в исследовании политического дискурса, Ф. Лейфелд предложил и успешно реализовал вариант заполнения его на основе новой методологии эмпирического и теоретического анализа политического дискурса посредством концепта дискурсивного сетевого анализа, связывающего каждого политического актора с его контентом, представляющего собой комбинацию качественного контент-анализа и количественного социально-сетевого анализа и призванного концептуализировать и замерять ко-эволюцию акторов и их контентов в процессе динамических изменений реформирования и смены политических курсов, что свидетельствует о его достаточном объяснительном потенциале и определяет его релевантность задаче исследования дискурсивно-динамического аспекта публичной политики. Этой же задаче соответствует и предложенная автором тонкая, многогранная интерпретация центрального в концепте дискурсивного сетевого анализа понятия политического дискурса, а также значительный уже опыт практического применения дискурсивного сетевого анализа в изучении различных проблем современной публичной политики.

\section{Список литературы}

1. Мирошниченко И. В., Морозова Е.В. 2017. Сетевая публичная политика: контуры предметного поля. Полис. Политические исследования, № 2: 82-102.

2. Atkinson R. 2000. Narratives of Policy: the Construction of Urban Problems and Urban Policy in the Official Discourse of British Government 1968-1998. Critical Social Policy, 20: 211-232.

3. Baumgartner F.R. and Jones B.D. (1991) Agenda dynamics and policy subsystems. The Journal of Politics, 53 (4): 1044-1074.

4. Berardo Ramiro and John T. Scholz. 2010. Self-Organizing Policy Networks: Risk, Partner Selection, and Cooperation in Estuaries. American Journal of Political Science, 54 (3): 632-649.

5. Berardo Ramiro and Mark Lubell. 2016. Understanding What Shapes a Polycentric Governance System. Public Administration Review, 76 (5): 738-751.

6. Berardo Ramiro. 2014. Bridging and Bonding Capital in Two-Mode Collaboration Networks. Policy Studies Journal, 42 (2): 197-225.

7. Brandes U. and Corman S.R. 2003. Visual unrolling of network evolution and the analysis of dynamic discourse. Information Visualization, 2 (1): 40-50.

8. Bryman A. 2012. Social Research Methods. Oxford: Oxford University Press: 766 p.

9. Clegg S.S. 1989. Frameworks of Power. London: Sage: 297 p.

10. Falk D. 2007. Policy Framing in the European Union. Journal of European Public Policy, 14 (4): 654-666.

11. Ferrari F. 2007. Metaphor at work in the analysis of political discourse: investigating a 'preventive war' persuasion strategy. Discourse \& Society, 18 (5): 603-625.

12. Fischer F., and J. Forester. (eds.) 1993. The argumentative turn in policy analysis and planning. Durham: Duke University Press: 352 p.

13. Ghinoi S. \& Steiner B. 2020. The political debate on climate change in Italy: A discourse network analysis. Politics and Governance, 8 (2): 215-228.

14. Haas P.M. 1992. Introduction: Epistemic communities and international policy coordination. International Organization, 46 (1): 1-35. 
15. Hajer M. 1993. Discourse Coalitions and the Institutionalization of Practice: The Case of Acid Rain in Britain. In: Fischer F. and J. Forester (eds.). The Argumentative Turn in Policy Analysis and Planning. Durham, NC: Duke University Press, pp. 34-76.

16. Hajer M. 1996. Ecological Modernization as Cultural Politics. In: Lash S., B. Szerszynski and B. Wynne, (eds.). Risk, Environment and Modernity: Towards a New Ecology. London: Sage: 246-268.

17. Hajer M. (2002) «Discourse Analysis and the Study of Policy Making», European Political Science 2 (1): 61-65.

18. Hall P.A. 1993. Policy paradigms, social learning, and the state: the case of economic policymaking in Britain. Comparative Politics, 25 (3): 275-296.

19. Haunss S., Kuhn J., Padó S., Blessing A., Blokker N., Dayanik E. \& Lapesa G. (2020) Integrating manual and automatic annotation for the creation of discourse network data sets. Politics and Governance, 8 (2): 326-339.

20. Howe A.C., M.C.J. Stoddart and D.B. Tindall. 2020. Media coverage and perceived policy influence of environmental actors: Good strategy or pyrrhic victory? Politics and Governance, 8 (2): 298-310.

21. Hurrelmann A., Krell-Laluhov Z., Nullmeier F., Schneider S. and Wiesner A. 2009. Why the democratic nation-state is still legitimate: A study of media discourses. European Journal of Political Research, 48 (4): 483-515.

22. Johnson D.W. and Johnson R.T. 2000. Civil political discourse in a democracy: The contribution of psychology. Peace and Conflict: Journal of Peace Psychology, 6 (4): 291-317.

23. Kukkonen A. and Ylä-Anttila T. 2020. The science-policy interface as a discourse network: Finland's climate change policy 2002-2015. Politics and Governance, 8 (2): 200-214.

24. Laclau E. and C. Mouffe. 1985. Hegemony and Socialist Strategy: Towards a Radical Democratic Politics. London: Verso: 240 p.

25. Leifeld P. 2010. Ideas, policy design and policy instruments: casting light on the missing link. Paper presented at the workshop of ECPR Joint Sessions, Munster, March 22-27, 2010. https://ecpr.eu/Filestore/PaperProposal/d9d2e2b4-306f-4bed-a1a9-8be194ff7dbf.pdf

26. Leifeld P. 2013. Reconceptualizing major policy change in the advocacy coalition framework: A discourse network analysis of German pension politics. Policy Studies Journal, 41 (1): 169-198.

27. Leifeld P. 2014. Polarization of coalitions in an agent-based model of political discourse. Computational Social Networks, 1 (1), article No. 7. https://doi.org/10.1186/s40649-014-0007-y.

28. Leifeld P. 2017. Discourse network analysis: Policy debates as dynamic networks. In: Victor J.N., A.H. Montgomery \& M.N. Lubell (Eds.). The Oxford handbook of political networks. Oxford: Oxford University Press: 301-325.

29. Leifeld P. and S. Haunss. 2012. Political Discourse Networks and the Conflict Over Software Patents in Europe. European Journal of Political Research, 51 (3): 382-409 doi: 10.1111/j.14756765.2011.02003.x.

30. Leifeld P. and L. Brandenberger. 2019. Endogenous coalition formation in policy debates. Retrieved from https://arxiv.org/abs/1904.05327.

31. Leifeld P. and S. Haunss. 2012. Political discourse networks and the conflict over software patents in Europe. European Journal of Political Research, 51 (3): 382-409.

32. Leifeld P. 2020. Policy Debates and Discourse Network Analysis: A Research Agenda. Politics and Governance, 8 (2): 180-183.

33. Lenschow A. and A.R. Zito. 1998. Blurring or Shifting of Policy Frames? Institutionalization of the Economic-Environmental Policy Linkage in the European Community. Governance: An International Journal of Policy and Administration, 11 (4): 415-441.

34. Linder S. 1995. Contending Discourses in the Electric and Magnetic Fields Controversy: The Social Construction of EMF Risk as a Public Problem, Policy Sciences, 28 (2): 209-230.

35. Muller Allan. 2015. Using Discourse Network Analysis to Measure Discourse Coalitions: Towards a Formal Analysis of Political Discourse. World Political Science. 11 (2): 377-404.

36. Popping R. 2003. Knowledge graphs and network text analysis. Social Science Information, 42 (1): 91-106.

37. Sabatier P.A. and C.M. Weible. 2007. The advocacy coalition framework. In: Sabatier P.A., (ed.) Theories of the Policy Process. Boudler CO: Westview Press: 189-222.

38. Sederberg P.C. 1984. The Politics of Meaning. Tucson, Arizona: The University of Arizona Press: 294 p. 
39. Schaub S. and Metz F. 2020. Comparing discourse and policy network approaches: Evidence from water policy on micropollutants. Politics and Governance, 8 (2): 184-199.

40. Szarka J. 2004. Wind Power, Discourse Coalitions and Climate Change: Breaking the Stalemate? European Environment, 14 (6): 314-330.

41. Victor J.N., Montgomery A.N. and Lubell M.N. (Eds.). 2017. The Oxford Handbook of Political Networks. Oxford: Oxford University Press: 1008 p.

42. Wallaschek S., Starke C. and Brüning C. 2020. Solidarity in the public sphere: A discourse network analysis of German newspapers (2008-2017). Politics and Governance, 8 (2): 257-271.

43. Weible C.M. and Sabatier P.A. (Eds.). 2017. Theories of the policy process (4th ed.). New York, NY: Routledge: 416 p.

44. Wodak R. and Meyer M. 2009. Critical discourse analysis: History, agenda, theory and methodology. In: Wodak R. and Meyer M., (eds.). Methods for Critical Discourse Analysis. London: Sage Publications Ltd: 1-33.

45. Yanow D. 2000. Conducting Interpretive Policy Analysis. London: Sage: 120 p.

\section{References}

1. Miroshnichenko I.V., Morozova E.V. 2017. Setevaya publichnaya politika: konturi predmetnogo polya [Network public policy: the subject field contours]. Polis. Politicheskiye issledovaniya. No. 2: 82-102.

2. Atkinson R. 2000. Narratives of Policy: the Construction of Urban Problems and Urban Policy in the Official Discourse of British Government 1968-1998. Critical Social Policy, 20: 211-232.

3. Baumgartner F.R. and Jones B.D. (1991) Agenda dynamics and policy subsystems. The Journal of Politics, 53 (4): 1044-1074.

4. Berardo Ramiro and John T. Scholz. 2010. Self-Organizing Policy Networks: Risk, Partner Selection, and Cooperation in Estuaries. American Journal of Political Science, 54 (3): 632-649.

5. Berardo Ramiro and Mark Lubell. 2016. Understanding What Shapes a Polycentric Governance System. Public Administration Review, 76 (5): 738-751.

6. Berardo Ramiro. 2014. Bridging and Bonding Capital in Two-Mode Collaboration Networks. Policy Studies Journal, 42 (2): 197-225.

7. Brandes U. and Corman S.R. 2003. Visual unrolling of network evolution and the analysis of dynamic discourse. Information Visualization, 2 (1): 40-50.

8. Bryman A. 2012. Social Research Methods. Oxford: Oxford University Press: 766 p.

9. Clegg S.S. 1989. Frameworks of Power. London: Sage: 297 p.

10. Falk D. 2007. Policy Framing in the European Union. Journal of European Public Policy, 14 (4): 654-666.

11. Ferrari F. 2007. Metaphor at work in the analysis of political discourse: investigating a 'preventive war' persuasion strategy. Discourse \& Society, 18 (5): 603-625.

12. Fischer F., and J. Forester. (eds.) 1993. The argumentative turn in policy analysis and planning. Durham: Duke University Press: 352 p.

13. Ghinoi S. \& Steiner B. 2020. The political debate on climate change in Italy: A discourse network analysis. Politics and Governance, 8 (2): 215-228.

14. Haas P.M. 1992. Introduction: Epistemic communities and international policy coordination. International Organization, 46 (1): 1-35.

15. Hajer M. 1993. Discourse Coalitions and the Institutionalization of Practice: The Case of Acid Rain in Britain. In: Fischer F. and J. Forester (eds.). The Argumentative Turn in Policy Analysis and Planning. Durham, NC: Duke University Press, pp. 34-76.

16. Hajer M. 1996. Ecological Modernization as Cultural Politics. In: Lash S., B. Szerszynski and B. Wynne, (eds.). Risk, Environment and Modernity: Towards a New Ecology. London: Sage: 246-268.

17. Hajer M. (2002) «Discourse Analysis and the Study of Policy Making», European Political Science 2 (1): 61-65.

18. Hall P.A. 1993. Policy paradigms, social learning, and the state: the case of economic policymaking in Britain. Comparative Politics, 25 (3): 275-296.

19. Haunss S., Kuhn J., Padó S., Blessing A., Blokker N., Dayanik E. \& Lapesa G. (2020) Integrating manual and automatic annotation for the creation of discourse network data sets. Politics and Governance, 8 (2): 326-339. 
20. Howe A.C., M.C.J. Stoddart and D.B. Tindall. 2020. Media coverage and perceived policy influence of environmental actors: Good strategy or pyrrhic victory? Politics and Governance, 8 (2): $298-310$.

21. Hurrelmann A., Krell-Laluhov Z., Nullmeier F., Schneider S. and Wiesner A. 2009. Why the democratic nation-state is still legitimate: A study of media discourses. European Journal of Political Research, 48 (4): 483-515.

22. Johnson D.W. and Johnson R.T. 2000. Civil political discourse in a democracy: The contribution of psychology. Peace and Conflict: Journal of Peace Psychology, 6 (4): 291-317.

23. Kukkonen A. and Ylä-Anttila T. 2020. The science-policy interface as a discourse network: Finland's climate change policy 2002-2015. Politics and Governance, 8 (2): 200-214.

24. Laclau E. and C. Mouffe. 1985. Hegemony and Socialist Strategy: Towards a Radical Democratic Politics. London: Verso: 240 p.

25. Leifeld P. 2010. Ideas, policy design and policy instruments: casting light on the missing link. Paper presented at the workshop of ECPR Joint Sessions, Munster, March 22-27, 2010. https://ecpr.eu/Filestore/PaperProposal/d9d2e2b4-306f-4bed-a1a9-8be194ff7dbf.pdf

26. Leifeld P. 2013. Reconceptualizing major policy change in the advocacy coalition framework: A discourse network analysis of German pension politics. Policy Studies Journal, 41 (1): 169-198.

27. Leifeld P. 2014. Polarization of coalitions in an agent-based model of political discourse. Computational Social Networks, 1 (1), article No. 7. https://doi.org/10.1186/s40649-014-0007-y.

28. Leifeld P. 2017. Discourse network analysis: Policy debates as dynamic networks. In: Victor J.N., A.H. Montgomery \& M.N. Lubell (Eds.). The Oxford handbook of political networks. Oxford: Oxford University Press: 301-325.

29. Leifeld P. and S. Haunss. 2012. Political Discourse Networks and the Conflict Over Software Patents in Europe. European Journal of Political Research, 51 (3): 382-409 doi: 10.1111/j.14756765.2011.02003.x.

30. Leifeld P. and L. Brandenberger. 2019. Endogenous coalition formation in policy debates. Retrieved from https://arxiv.org/abs/1904.05327.

31. Leifeld P. and S. Haunss. 2012. Political discourse networks and the conflict over software patents in Europe. European Journal of Political Research, 51 (3): 382-409.

32. Leifeld P. 2020. Policy Debates and Discourse Network Analysis: A Research Agenda. Politics and Governance, 8 (2): 180-183.

33. Lenschow A. and A.R. Zito. 1998. Blurring or Shifting of Policy Frames? Institutionalization of the Economic-Environmental Policy Linkage in the European Community. Governance: An International Journal of Policy and Administration, 11 (4): 415-441.

34. Linder S. 1995. Contending Discourses in the Electric and Magnetic Fields Controversy: The Social Construction of EMF Risk as a Public Problem, Policy Sciences, 28 (2): 209-230.

35. Muller Allan. 2015. Using Discourse Network Analysis to Measure Discourse Coalitions: Towards a Formal Analysis of Political Discourse. World Political Science. 11 (2): 377-404.

36. Popping R. 2003. Knowledge graphs and network text analysis. Social Science Information, 42 (1): 91-106.

37. Sabatier P.A. and C.M. Weible. 2007. The advocacy coalition framework. In: Sabatier P.A., (ed.) Theories of the Policy Process. Boudler CO: Westview Press: 189-222.

38. Sederberg P.C. 1984. The Politics of Meaning. Tucson, Arizona: The University of Arizona Press: 294 p.

39. Schaub S. and Metz F. 2020. Comparing discourse and policy network approaches: Evidence from water policy on micropollutants. Politics and Governance, 8 (2): 184-199.

40. Szarka J. 2004. Wind Power, Discourse Coalitions and Climate Change: Breaking the Stalemate? European Environment, 14 (6): 314-330.

41. Victor J.N., Montgomery A.N. and Lubell M.N. (Eds.). 2017. The Oxford Handbook of Political Networks. Oxford: Oxford University Press: 1008 p.

42. Wallaschek S., Starke C. and Brüning C. 2020. Solidarity in the public sphere: A discourse network analysis of German newspapers (2008-2017). Politics and Governance, 8 (2): 257-271.

43. Weible C.M. and Sabatier P.A. (Eds.). 2017. Theories of the policy process (4th ed.). New York, NY: Routledge: 416 p. 
44. Wodak R. and Meyer M. 2009. Critical discourse analysis: History, agenda, theory and methodology. In: Wodak R. and Meyer M., (eds.). Methods for Critical Discourse Analysis. London: Sage Publications Ltd: 1-33.

45. Yanow D. 2000. Conducting Interpretive Policy Analysis. London: Sage: 120 p.

ИНФОРМАЦИЯ ОБ АВТОРЕ

Корюшкин Александр Иванович, доцент кафедры теории и философии политики СанктПетербургского государственного университета, г. Санкт-Петербург, Россия

\section{INFORMATION ABOUT THE AUTHOR}

Alexander I. Koryushkin, associate professor of the department of theory and philosophy of politics of St. Petersburg State University, St. Petersburg, Russia 